\title{
THE AREA OF A SPECTRALLY POSITIVE STABLE PROCESS STOPPED AT ZERO
}

\author{
BY
}

JULIEN LETEMPLIER (LILLE) AND THOMAS SIMON (LILLE)

Abstract. A multiplicative identity in law for the area of a spectrally positive Lévy $\alpha$-stable process stopped at zero is established. Extending that of Lefebvre for Brownian motion, it involves an inverse beta random variable and the square of a positive stable random variable. This simple identity makes it possible to study precisely the behaviour of the density at zero, which is Fréchet-like.

2010 AMS Mathematics Subject Classification: Primary: 60G52; Secondary: 60E07, 60G51.

Key words and phrases: Hitting time, integrated process, stable Lévy process, tail asymptotics.

\section{INTRODUCTION AND STATEMENT OF THE RESULTS}

Let $\left\{B_{t}, t \geqslant 0\right\}$ be a linear Brownian motion, starting from $B_{0}=1$. Denote its first hitting time of zero by $T=\inf \left\{t>0, B_{t}=0\right\}$. The random variable

$$
\mathcal{A}=\int_{0}^{T} B_{s} d s
$$

has been investigated by Lefebvre, who obtained in Theorem 2 of [1]] the identity in law

$$
\mathcal{A} \stackrel{d}{=} \frac{2}{9 \Gamma_{1 / 3}},
$$

where, here and throughout, $\boldsymbol{\Gamma}_{a}$ stands for the standard gamma random variable with density

$$
\frac{x^{a-1}}{\Gamma(a)} e^{-x} \mathbf{1}_{\{x>0\}} .
$$

The identity (III) is obtained as a consequence of the closed expression for the Laplace transform of the bivariate random variable $(T, \mathcal{A})$ in terms of the Airy 
function - see Theorem 1 in [11]. As observed in [10], p. 402, this latter expression can be easily derived thanks to the Feynman-Kac formula. Notice that Airy functions appear in the expression of the Laplace transform of many other Brownian areas (see [6]), whose laws are in general more complicated than (ID. I). It is also interesting to mention that the random variable on the right-hand side of (II.]) can also be viewed as a Brownian perpetuity, according to Dufresne's celebrated identity [4].

In a recent paper [12], we have generalized (IDI) to random variables of the type

$$
\int_{0}^{T}\left(L_{s}\right)^{q} d s, \quad q \in \mathbb{R},
$$

where $L$ is a Lévy strictly stable process starting from one, and $T$ is its first passage time below zero. A general identity was obtained involving infinite independent products of beta random variables, and several distributional properties related to infinite divisibility were deduced - see [12] for details. However, this identity remains complicated, and the corresponding expression of the Mellin transform, given in [12] in terms of the double gamma function, can be difficult to invert in order to get tail asymptotics of the density.

In this note, we derive a very simple identity for the particular random variable

$$
\mathcal{A}_{\alpha}=\int_{0}^{T} L_{s}^{(\alpha)} d s,
$$

where $\left\{L_{t}^{(\alpha)}, t \geqslant 0\right\}$ is a strictly $\alpha$-stable Lévy process without negative jumps, starting from one, and $T=\inf \left\{t>0, L_{t}^{(\alpha)}=0\right\}$ is its first hitting time of zero. Without loss of generality we choose the normalization

$$
\mathbb{E}\left[e^{-t L_{1}^{(\alpha)}}\right]=e^{t^{\alpha}}, \quad t \geqslant 0,
$$

where $\alpha \in[1,2]$ is the self-similarity parameter. We refer, e.g., to Chapter 3 in [15] for more information on stable Lévy processes and the above normalization. The boundary cases $\alpha=1,2$ correspond, respectively, to the unit drift and the Brownian motion with variance $\sqrt{2}$, so that we have

$$
\mathcal{A}_{1}=\frac{1}{2} \quad \text { and } \quad \mathcal{A}_{2} \stackrel{d}{=} \frac{1}{9 \Gamma_{1 / 3}} .
$$

The second identity in (․2), which is actually the precise statement of Theorem 2 in [I]], follows from (II) and the self-similarity of Brownian motion. In order to state our result, we need some further notation. Introduce the beta random variable $\mathbf{B}_{a, b}$ with density

$$
\frac{\Gamma(a+b)}{\Gamma(a) \Gamma(b)} x^{a-1}(1-x)^{b-1} \mathbf{1}_{(0,1)}(x),
$$


and the positive $a$-stable random variable $\mathbf{Z}_{a}$ with Laplace transform

$$
\mathbb{E}\left[e^{-\lambda \mathbf{Z}_{a}}\right]=e^{-\lambda^{a}}, \quad \lambda \geqslant 0 .
$$

THEOREM 1.1. For every $\alpha \in(1,2)$, one has the independent factorization

$$
\mathcal{A}_{\alpha} \stackrel{d}{=}\left(\frac{\alpha+1}{4}\right) \times \mathbf{Z}_{\frac{2}{\alpha+1}}^{2} \times \mathbf{B}_{\frac{1}{2}, \frac{\alpha-1}{2(\alpha+1)}}^{-1} .
$$

Observe that ([L.3) is in accordance with the two boundary cases: when $\alpha=1$, the two random variables on the right-hand side boil down to one, whereas when $\alpha=2$, the following identity obtained in Theorem 1 of [17],

$$
\mathbf{Z}_{2 / 3}^{2} \stackrel{d}{=} \frac{4}{27} \boldsymbol{\Gamma}_{2 / 3}^{-1} \times \mathbf{B}_{1 / 3,1 / 6}^{-1}
$$

combined with the elementary factorization $\boldsymbol{\Gamma}_{a} \stackrel{d}{=} \boldsymbol{\Gamma}_{a+b} \times \mathbf{B}_{a, b}$, allows us to recover the second identity in (․2.). As in [12], the proof of ([1.3) relies on an identification of the fractional moments of $\mathcal{A}_{\alpha}$ in terms of the gamma function, see (2.]). However, the method of [12] is based on a modern and rather heavy machinery involving the Lamperti transform and exponential functional of Lévy processes, whereas our argument here is easier and more classical, using the strong Markov property and some exact results on the stable Kolmogorov process recently obtained in [14].

The simple expression of the Mellin transform of $\mathcal{A}_{\alpha}$ can be easily inverted in order to give a convergent series representation for its density $f_{\mathcal{A}_{\alpha}}$. Throughout, we will set $f_{X}$ for the density of an absolutely continuous random variable $X$.

COROLLARY 1.1. The density of $\mathcal{A}_{\alpha}$ has a convergent series representation:

$$
f_{\mathcal{A}_{\alpha}}(x)=\Gamma\left(\frac{\alpha}{\alpha+1}\right) \times \sum_{n=0}^{\infty} \frac{(-1)^{n}(\alpha+1)^{\frac{n+1}{\alpha+1}-1} x^{-\frac{n+1}{\alpha+1}-1}}{n ! \Gamma\left(1-\frac{n+1}{\alpha+1}\right) \Gamma\left(1-\frac{n+2}{\alpha+1}\right)}, \quad x>0 .
$$

Observe that when $\alpha=2$, the above summation is made over $n=3 p$ only, and that further simplifications lead to

$$
f_{\mathcal{A}_{2}}(x)=\frac{x^{-4 / 3}}{3^{2 / 3} \Gamma(1 / 3)} \sum_{p=0}^{\infty} \frac{(-1)^{p}(9 x)^{-p}}{p !}=\frac{\Gamma(2 / 3) x^{-4 / 3} e^{-1 /(9 x)}}{2 \pi 3^{1 / 6}},
$$

which is precisely the expression to be found in Theorem 2 of [11]. Corollary 1.1 also implies the first order asymptotic behaviour:

$$
f_{\mathcal{A}_{\alpha}}(x) \sim \frac{(\alpha+1)^{\frac{1}{\alpha+1}-1} x^{-\frac{1}{\alpha+1}-1}}{\Gamma\left(\frac{\alpha-1}{\alpha+1}\right)}, \quad x \rightarrow \infty .
$$


This behaviour has, up to a multiplicative constant, the same speed as that of the density of the factor $\mathbf{Z}_{2 /(\alpha+1)}^{2}$ at infinity - see formula (14.31) in [15].

On the other hand, it does not seem possible to find a suitable transform of the above series representation in order to deduce the exact behaviour of $f_{\mathcal{A}_{\alpha}}$ at zero. Usually this is done via the Mellin-Barnes transform and the residue theorem, but here this method fails because $\mathcal{A}_{\alpha}$ has negative moments of every order. Using (ㅍ.3), we can show the following precise estimate.

COROLLARY 1.2. The asymptotic behaviour of the density of $\mathcal{A}_{\alpha}$ as $x \rightarrow 0+$ is given by

$$
f_{\mathcal{A}_{\alpha}}(x) \sim \kappa_{\alpha} x^{\alpha^{2} /\left(1-\alpha^{2}\right)} \exp \left(-c_{\alpha} x^{1 /(1-\alpha)}\right),
$$

with

$$
\kappa_{\alpha}=\frac{\Gamma\left(\frac{\alpha}{\alpha+1}\right) \sqrt{\frac{\alpha+1}{\alpha-1}}}{2 \pi(\alpha+1)^{\alpha /\left(\alpha^{2}-1\right)}} \quad \text { and } \quad c_{\alpha}=(\alpha-1)(\alpha+1)^{\alpha /(1-\alpha)} .
$$

This shows that the behaviour of $f_{\mathcal{A}_{\alpha}}$ at zero is that of the generalized Fréchet density

$$
f_{c_{\alpha}^{\alpha-1} \boldsymbol{\Gamma}_{1 /(\alpha+1)}^{1-\alpha}}(x)=\tilde{\kappa}_{\alpha} x^{\alpha^{2} /\left(1-\alpha^{2}\right)} \exp \left(-c_{\alpha} x^{1 /(1-\alpha)}\right),
$$

up to the normalizing constant

$$
\tilde{\kappa}_{\alpha}=\frac{(\alpha-1)^{-\alpha /(\alpha+1)}}{\Gamma\left(\frac{1}{\alpha+1}\right)(\alpha+1)^{\alpha /\left(\alpha^{2}-1\right)}}
$$

which does not coincide with $\kappa_{\alpha}$ except for $\alpha=2$. Observe also that making $\alpha=2$ on the right-hand side of ([1.5) yields the density in (IL.4). Using our method, it should be possible to obtain a full asymptotic expansion of $f_{\mathcal{A}_{\alpha}}$ at zero, in the spirit of (14.35) in [15] - see Remark 2.2 below. But we have not adressed this question, which is believed to be rather technical, in the present note.

\section{PROOFS}

2.1. Proof of Theorem 1.1. To simplify the notation, we will set $L=L^{(\alpha)}$. Introduce the area process

$$
A_{t}=\int_{0}^{t} L_{s} d s, \quad t \geqslant 0 .
$$

Recall that the bivariate process $X=\left\{\left(A_{t}, L_{t}\right), t \geqslant 0\right\}$ is strongly Markovian and denote by $\mathbb{P}_{(x, y)}$ its law starting from $(x, y)$. Consider the stopping time

$$
S=\inf \left\{t>0, A_{t}=0\right\}
$$

and observe that under $\mathbb{P}_{(0,1)}$ one has a.s. $S>T, A_{T}>0$, and $L_{S}<0$. 
Setting $\left\{\mathcal{F}_{t}, t \geqslant 0\right\}$ for the natural completed filtration of $X$ and applying the strong Markov property at $T$ shows that for every $s \in \mathbb{R}$ one has

$$
\begin{aligned}
\mathbb{E}_{(0,1)}\left[\left|L_{S}\right|^{s-1}\right]=\mathbb{E}_{(0,1)}\left[\mathbb{E}\left[\left|L_{S}\right|^{s-1} \mid \mathcal{F}_{T}\right]\right] & =\mathbb{E}_{(0,1)}\left[\mathbb{E}_{\left(\mathcal{A}_{\alpha}, 0\right)}\left[\left|L_{S}\right|^{s-1}\right]\right] \\
& =\mathbb{E}\left[\mathcal{A}_{\alpha}^{(s-1) /(\alpha+1)}\right] \times \mathbb{E}_{(1,0)}\left[\left|L_{S}\right|^{s-1}\right],
\end{aligned}
$$

possibly with infinite values on both sides, where the second equality follows from the absence of negative jumps for $L$, and the third equality from the self-similarity of $L$ and $A$ with respective indices $1 / \alpha$ and $1+1 / \alpha$, which readily implies that the law of $L_{S}$ under $\mathbb{P}_{(x, 0)}$ is that of $x^{1 /(\alpha+1)} L_{S}$ under $\mathbb{P}_{(1,0)}$ for every $x>0$.

Applying now Theorem B of [14] in the particular case $\rho=1 / \alpha$ (beware that we consider here the dual process, with no positive jumps), we get

$$
\mathbb{E}_{(0,1)}\left[\left|L_{S}\right|^{s-1}\right]=\frac{\sin \left(\frac{\pi s}{\alpha+1}\right)}{\sin \left(\frac{\pi \alpha s}{\alpha+1}\right)}
$$

and

$$
\mathbb{E}_{(1,0)}\left[\left|L_{S}\right|^{s-1}\right]=\frac{(\alpha+1)^{(1-s) /(\alpha+1)} \Gamma\left(\frac{\alpha+2}{\alpha+1}\right) \Gamma\left(\frac{1-s}{\alpha+1}\right) \sin \left(\frac{\pi}{\alpha+1}\right)}{\Gamma\left(\frac{s}{\alpha+1}\right) \Gamma(1-s) \sin \left(\frac{\pi \alpha s}{\alpha+1}\right)}
$$

for all $|s|<1+1 / \alpha$. Dividing and simplifying with the help of the complement formula for the gamma function, we deduce

$$
\mathbb{E}\left[\mathcal{A}_{\alpha}^{s}\right]=(\alpha+1)^{s} \times \frac{\Gamma\left(\frac{\alpha}{\alpha+1}\right) \Gamma(1-(\alpha+1) s)}{\Gamma\left(\frac{\alpha}{\alpha+1}-s\right) \Gamma(1-s)}
$$

for all $s<1 /(\alpha+1)$. Applying Gauss's multiplication formula for the gamma function (see, e.g., Theorem 1.5.1 in [1] ) implies

$$
\mathbb{E}\left[\mathcal{A}_{\alpha}^{s}\right]=\left(\frac{\alpha+1}{4}\right)^{s} \times \frac{\Gamma(1-(\alpha+1) s)}{\Gamma(1-2 s)} \times \frac{\Gamma\left(\frac{\alpha}{\alpha+1}\right) \Gamma\left(\frac{1}{2}-s\right)}{\Gamma\left(\frac{1}{2}\right) \Gamma\left(\frac{\alpha}{\alpha+1}-s\right)},
$$

and we can conclude by a fractional moment identification, recalling (see, e.g., formula (25.5) in [15] for the second expression) that

$$
\mathbb{E}\left[\mathbf{B}_{a, b}^{s}\right]=\frac{\Gamma(a+s) \Gamma(a+b)}{\Gamma(a) \Gamma(a+b+s)} \quad \text { and } \quad \mathbb{E}\left[\mathbf{Z}_{a}^{s}\right]=\frac{\Gamma\left(1-\frac{s}{a}\right)}{\Gamma(1-s)} .
$$

REMARK 2.1. (a) With the notation of our proof above, it is possible to derive the law of $A_{T}$ under $\mathbb{P}_{(x, y)}$ for any $x \in \mathbb{R}$ and $y>0$, by the self-similarity of $L^{(\alpha)}$. One finds

$$
\mathcal{A}_{\alpha} \stackrel{d}{=} x+\left(\frac{(\alpha+1) y^{\alpha+1}}{4}\right) \times \mathbf{Z}_{\frac{2}{\alpha+1}}^{2} \times \mathbf{B}_{\frac{1}{2}, \frac{\alpha-1}{2(\alpha+1)}}^{-1} .
$$


(b) It is well known and easy to verify (see, e.g., Theorem 46.3 in [15]) that under $\mathbb{P}_{(0,1)}$, the random variable $T$ is distributed as $\mathbf{Z}_{1 / \alpha}$. The theorem above provides a connection between $\mathcal{A}_{\alpha}=A_{T}$ and the random variable $\mathbf{Z}_{2 /(\alpha+1)}$. However, it seems difficult with our method to obtain some valuable information on the Mellin transform of the bivariate random variable $\left(T, A_{T}\right)$.

(c) Using again Gauss's multiplication formula, it is possible to derive from (2.]) the other independent factorization,

$$
\mathcal{A}_{\alpha} \stackrel{d}{=}(\alpha+1)^{-1} \times \mathbf{Z}_{\frac{2}{\alpha+1}} \times \mathbf{Z}_{\frac{2}{\alpha+1}}^{\left(\frac{1}{\alpha+1}\right)},
$$

where $X^{(t)}$ is the size bias of order $t$ of a positive random variable $X$, that is,

$$
\mathbb{E}\left[f\left(X^{(t)}\right)\right]=\frac{\mathbb{E}\left[X^{t} f(X)\right]}{\mathbb{E}\left[X^{t}\right]}
$$

for every $f$ bounded continuous. This factorization makes the connection between $\mathcal{A}_{\alpha}$ and $\mathbf{Z}_{2 /(\alpha+1)}$ even tighter, but it is less tractable to obtain Corollary 1.2.

(d) Combining the Kanter factorization (see Corollary 4.1 in [7]) and ([L.3]) shows the identity

$$
\mathcal{A}_{\alpha} \stackrel{d}{=}\left(\frac{\alpha+1}{4}\right) \times \boldsymbol{\Gamma}_{1}^{1-\alpha} \times\left(\mathbf{B}_{\frac{1}{2}, \frac{\alpha-1}{2(\alpha+1)}} \times \mathbf{K}_{\frac{2}{\alpha+1}}^{\alpha+1}\right)^{-1},
$$

where $\mathbf{K}_{a}$ is the so-called Kanter random variable of index $a \in(0,1)$ - see Section 3 in [117] for details. Moreover, the observations made in Remark 3.1 of [9] show that

$$
\int_{0}^{T}\left(L_{t}^{(\alpha)}\right)^{-1} d t \stackrel{d}{=} \frac{1}{(\alpha-1) \Gamma_{1}^{\alpha-1}}
$$

We can then deduce the curious factorization

$$
\int_{0}^{T} L_{t}^{(\alpha)} d t \stackrel{d}{=}\left(\frac{\alpha^{2}-1}{4}\right) \times\left(\mathbf{B}_{\frac{1}{2}, \frac{\alpha-1}{2(\alpha+1)}} \times \mathbf{K}_{\frac{2}{\alpha+1}}^{\alpha+1}\right)^{-1} \times\left(\int_{0}^{T}\left(L_{t}^{(\alpha)}\right)^{-1} d t\right) .
$$

2.2. Proof of Corollary 1.1. We will reason along the same lines as in Proposition 2 in [16], and omit some details. Applying the Mellin inversion formula yields first

$$
f_{\mathcal{A}_{\alpha}}(x)=\frac{1}{2 \pi x} \int_{\mathbb{R}} \mathcal{M}_{\alpha}(s) x^{-\mathrm{i} s} d s,
$$

with the notation

$$
\mathcal{M}_{\alpha}(s)=\mathbb{E}\left[\mathcal{A}_{\alpha}^{\mathrm{i} s}\right]=(\alpha+1)^{\mathrm{i} s} \times \frac{\Gamma\left(\frac{\alpha}{\alpha+1}\right) \Gamma(1-(\alpha+1) \mathrm{i} s)}{\Gamma\left(\frac{\alpha}{\alpha+1}-\mathrm{i} s\right) \Gamma(1-\mathrm{i} s)}
$$


for every $s \in \mathbb{R}$. Observe that the integral in the above-given formula converges for all $x \in \mathbb{C}$ with $\arg (x)<\pi(\alpha-1) / 2$, which can be seen from Stirling's asymptotics for the gamma function (see, e.g., Corollary 1.4.4 in [U]]). In particular, the density function $f_{\mathcal{A}_{\alpha}}$ is real analytic on $(0, \infty)$.

Suppose now $x>1$. We evaluate the integral above with the help of the residue theorem applied to the contour joining $-R$ to $R$ along the real axis, and $R$ to $-R$ along the half-circle plotted in the lower half-plane. It is easy to see that the integral along this half-circle vanishes as $R \rightarrow+\infty$, so that it remains to consider the singularities inside the contour, which are located at $t_{n}=-\mathrm{i}(n+1) /(\alpha+1)$, $n \geqslant 0$. Computing

$$
\operatorname{Res}_{t_{n}}\left(\mathcal{M}_{\alpha}(s) x^{-\mathrm{i} s}\right)=-\mathrm{i} \Gamma\left(\frac{\alpha}{\alpha+1}\right) \times \frac{(-1)^{n}(\alpha+1)^{\frac{n+1}{\alpha+1}-1} x^{-\frac{n+1}{\alpha+1}}}{n ! \Gamma\left(1-\frac{n+1}{\alpha+1}\right) \Gamma\left(1-\frac{n+2}{\alpha+1}\right)},
$$

we obtain

$$
f_{\mathcal{A}_{\alpha}}(x)=\Gamma\left(\frac{\alpha}{\alpha+1}\right) \times \sum_{n=0}^{\infty} \frac{(-1)^{n}(\alpha+1)^{\frac{n+1}{\alpha+1}-1} x^{-\frac{n+1}{\alpha+1}-1}}{n ! \Gamma\left(1-\frac{n+1}{\alpha+1}\right) \Gamma\left(1-\frac{n+2}{\alpha+1}\right)}
$$

for every $x>1$, and hence for every $x>0$ by analytic continuation (Stirling's formula shows indeed that the series on the right-hand side converges absolutely for every $x>0$, too).

REMARK 2.2. The results of [12] show the following representation of $\mathcal{A}_{\alpha}$ as a perpetuity. One has

$$
\mathcal{A}_{\alpha} \stackrel{d}{=} \int_{0}^{\infty} e^{-(\alpha+1) Z_{t}^{(\alpha)}} d t
$$

where $Z^{(\alpha)}$ is a spectrally negative Lévy process with Laplace exponent

$$
\log \mathbb{E}\left[e^{s Z_{1}^{(\alpha)}}\right]=\frac{\Gamma(\alpha+s)}{\Gamma(s)} .
$$

This Lévy process is of the hypergeometric type, with the terminology of [8]. However, the very general results of Section 4 in [8] do not cover our case. Observe also that our argument is straightforward and does not depend on the rational character of $\alpha$, as is the case for some results of [8].

2.3. Proof of Corollary 1.2. In order to simplify the notation, we will work on the random variable $\mathcal{X}_{\alpha}=\mathcal{A}_{\alpha}^{1 /(1-\alpha)}$. Changing the variable, we get the required estimate in the form

$$
f_{\mathcal{X}_{\alpha}}(x) \sim \frac{\Gamma\left(\frac{\alpha}{\alpha+1}\right) \sqrt{\alpha^{2}-1}}{2 \pi(\alpha+1)^{\alpha /\left(\alpha^{2}-1\right)}} x^{-\alpha /(\alpha+1)} e^{-c_{\alpha} x}, \quad x \rightarrow 0+.
$$


Evaluating with (2. I) the positive entire moments

$$
\mathbb{E}\left[\mathcal{X}_{\alpha}^{n}\right]=(\alpha+1)^{n /(1-\alpha)} \times \frac{\Gamma\left(\frac{\alpha}{\alpha+1}\right)}{\Gamma\left(\frac{\alpha}{\alpha+1}+\frac{n}{\alpha-1}\right)} \times \frac{\Gamma\left(1+\frac{(\alpha+1) n}{\alpha-1}\right)}{\Gamma\left(1+\frac{n}{\alpha-1}\right)}
$$

for every $n \geqslant 0$, and applying Stirling's formula, we obtain

$$
\frac{\left(\mathbb{E}\left[\mathcal{X}_{\alpha}^{n}\right]\right)^{1 / n}}{n} \rightarrow \frac{(\alpha+1)^{\alpha /(\alpha-1)}}{e(\alpha-1)} \text { as } n \rightarrow \infty .
$$

By a theorem of Davies-Kasahara (see Corollary 4.12.5 in [2], or Lemma 3.2 in [3] for a more appropriate formulation), we deduce that

$$
x^{-1} \log \mathbb{P}\left[\mathcal{X}_{\alpha}>x\right] \rightarrow-c_{\alpha} \quad \text { as } x \rightarrow+\infty .
$$

This yields the required asymptotic behaviour, at the logarithmic scale, for the survival function of $\mathcal{X}_{\alpha}$. Moreover, writing down, via Fubini's theorem, the moment generating function

$$
\mathbb{E}\left[e^{x \mathcal{X}_{\alpha}}\right]=\sum_{n=0}^{\infty} a_{n} x^{n}, \quad x>0,
$$

with

$$
\begin{aligned}
a_{n} & =(\alpha+1)^{n /(1-\alpha)} \times \frac{\Gamma\left(\frac{\alpha}{\alpha+1}\right) \Gamma\left(1+\frac{(\alpha+1) n}{\alpha-1}\right)}{n ! \Gamma\left(\frac{\alpha}{\alpha+1}+\frac{n}{\alpha-1}\right) \Gamma\left(1+\frac{n}{\alpha-1}\right)} \\
& \sim \frac{\Gamma\left(\frac{\alpha}{\alpha+1}\right) \sqrt{\alpha^{2}-1}}{2 \pi(\alpha-1)^{1 /(\alpha+1)}} c_{\alpha}^{-n} n^{-\alpha /(\alpha+1)} \quad \text { as } n \rightarrow \infty,
\end{aligned}
$$

and applying Karamata's theorem for power series (see Corollary 1.7.3 in [2]), we obtain

$$
\mathbb{E}\left[e^{c_{\alpha} x \mathcal{X}_{\alpha}}\right] \sim \frac{\Gamma\left(\frac{1}{\alpha+1}\right) \Gamma\left(\frac{\alpha}{\alpha+1}\right) \sqrt{\alpha^{2}-1}}{2 \pi(\alpha-1)^{1 /(\alpha+1)}(1-x)^{1 /(\alpha+1)}} \quad \text { as } x \rightarrow 1-.
$$

At this stage, it is worth mentioning that (2.4) can be obtained from (2.3]) by integration. However, it does not seem that we can infer the reverse inclusion without any further assumption, such as the existence of a meromorphic extension in the neighbourhood of $c_{\alpha}$ for the moment generating function (see Theorem 4 in [5]), or a monotonicity condition on $f_{\mathcal{X}_{\alpha}}$ at infinity (see Theorem 4.12.11 in [2]), which we both could not prove $a$ priori. 
In order to show (2.3) rigorously and complete the proof, we will use the following power transformation of (ㄴ.3):

$$
\mathcal{X}_{\alpha} \stackrel{d}{=}\left(\frac{4}{\alpha+1}\right)^{\frac{1}{\alpha-1}} \times \mathbf{B}_{\frac{1}{2}, \frac{\alpha-1}{2(\alpha+1)}}^{\frac{1}{\alpha-1}} \times \mathbf{Z}_{\frac{2}{\alpha+1}}^{\frac{2}{1-\alpha}} .
$$

The multiplicative convolution formula and a change of variable imply

$$
f_{\mathcal{X}_{\alpha}}(x)=\int_{0}^{\infty}\left(\frac{1}{1+y}\right) f_{\mathbf{U}_{\alpha}}\left(\frac{1}{1+y}\right) f_{\mathbf{V}_{\alpha}}(x+x y) d y
$$

where we have set

$$
\mathbf{U}_{\alpha}=\mathbf{B}_{\frac{1}{2}, \frac{\alpha-1}{2(\alpha+1)}}^{\frac{1}{\alpha-1}} \quad \text { and } \quad \mathbf{V}_{\alpha}=\left(\frac{4}{\alpha+1}\right)^{\frac{1}{\alpha-1}} \times \mathbf{Z}_{\frac{2}{\alpha+1}}^{\frac{2}{1-\alpha}} .
$$

On the one hand, we have

$$
\left(\frac{1}{1+y}\right) f_{\mathbf{U}_{\alpha}}\left(\frac{1}{1+y}\right)=\frac{(\alpha-1)^{\frac{\alpha-1}{2(\alpha+1)}} \Gamma\left(\frac{\alpha}{\alpha+1}\right)}{\sqrt{\pi} \Gamma\left(\frac{\alpha-1}{2(\alpha+1)}\right)} y^{\frac{\alpha-1}{2(\alpha+1)}-1}(1+O(y))
$$

as $y \rightarrow 0+$. On the other hand, formula (14.35) in [15] implies after, a change of variable and several simplifications,

$$
f_{\mathbf{V}_{\alpha}}(z)=\frac{\sqrt{\alpha-1}(\alpha+1)^{\frac{\alpha^{2}}{2\left(\alpha^{2}-1\right)}-\frac{1}{\alpha-1}}}{2 \sqrt{\pi}} z^{-1 / 2} e^{-c_{\alpha} z}\left(1+O\left(z^{-1 / 2}\right)\right)
$$

as $z \rightarrow+\infty$. Plugging these two first-order expansions in the integral (2.5), and making further simplifications, we finally get the required asymptotic behaviour (2.3).

\section{FINAL REMARKS}

3.1. On the infinite divisibility of $\mathcal{A}_{\alpha}$. From (2.2) or ([L.3), it is possible to derive several properties of $\mathcal{A}_{\alpha}$ related to infinite divisibility - see [112] for details, in particular Corollary 8 therein. It is interesting to mention that the sole infinite divisibility of $\mathcal{A}_{\alpha}$ can also be obtained by a very simple pathwise argument neither relying on (2.2) nor on ([L.3). Setting $T_{x}=\inf \left\{t>0, L_{t}=x\right\}$ for all $x>0$ and using the fact that $L$ has no negative jumps, it is easy to see from the Markov property that under $\mathbb{P}_{(0,1)}$, for every $n \geqslant 2$, there is an independent decomposition

$$
\mathcal{A}_{\alpha}=X_{1}^{(n)}+\ldots+X_{n}^{(n)},
$$


where

$$
X_{i}^{(n) \stackrel{d}{=}} A_{T_{\frac{n-i}{n}}} \text { under } \mathbb{P}_{\left(0, \frac{n+1-i}{n}\right)}
$$

for every $i=1, \ldots, n$. Moreover, one has $T_{1-1 / n} \rightarrow 0$ a.s. under $\mathbb{P}_{(0,1)}$ as $n \rightarrow$ $+\infty$ (by the well-known fact - see, e.g., Theorem 47.1 in [15] - that $L$ visits immediately the negative half-line when starting from zero), so that $X_{1}^{(n)} \rightarrow 0$ a.s. under $\mathbb{P}_{(0,1)}$ when $n \rightarrow+\infty$ as well. Last, it is straightforward that

$$
\mathbb{P}\left[\left|X_{i}^{(n)}\right|>\varepsilon\right] \leqslant \mathbb{P}\left[\left|X_{1}^{(n)}\right|>\varepsilon\right]
$$

for every $\varepsilon>0$ and $i=1, \ldots, n$. Putting everything together and applying Khinchin's theorem on triangular arrays (see, e.g., Theorem 9.3 in [15]) shows that $\mathcal{A}_{\alpha}$ is infinitely divisible.

Observe that the argument above does not make use of the self-similarity of $L$, and hence applies to any spectrally positive Lévy process which is not a subordinator.

3.2. Relation with other tail asymptotics. It is noticeable that the convolution formula (2.5) makes it possible to study the precise tail behaviour of other random variables defined as independent products. If we consider, for example,

$$
X=\mathbf{B}_{a, b}^{p} \times \boldsymbol{\Gamma}_{c}
$$

with $a, b, c, p>0$, this formula shows very quickly that

$$
f_{X}(x) \sim \frac{\Gamma(a+b)}{\Gamma(a) \Gamma(b)} p^{-b} x^{c / p-b-1} e^{-x}
$$

as $x \rightarrow+\infty$. At the less precise level of survival functions, this behaviour was recently obtained in [13], with a more complicated method. In this example it is also possible to derive without pain, contrary to that of $f_{\mathcal{X}_{\alpha}}(x)$, the full asymptotic expansion of $f_{X}(x)$ at infinity, which is finite for certain values of the parameters $a, b, c, p$.

Acknowledgments. We are grateful to the referee for having corrected a mistake in the original proof of Corollary 1.1 .

\section{REFERENCES}

[1] G. E. Andrews, R. Askey, and R. Roy, Special Functions, Cambridge University Press, Cambridge 1999.

[2] N. H. Bingham, C. M. Goldie, and J. L. Teugels, Regular Variation, Cambridge University Press, Cambridge 1987.

[3] M. Csörgö, Z. Shi, and M. Yor, Some asymptotic properties of the local time of the uniform empirical process, Bernoulli 5 (6) (1999), pp. 1035-1058.

[4] D. Dufresne, The distribution of a perpetuity, with applications to risk theory and pension funding, Scand. Actuar. J. 1 (1990), pp. 39-79. 
[5] P. Flajolet, X. Gourdon, and P. Dumas, Mellin transforms and asymptotics: Harmonic sums, Theoret. Comput. Sci. 144 (1995), pp. 3-58.

[6] S. Janson, Brownian excursion area, Wright's constants in graph enumeration, and other Brownian areas, Probab. Surv. 4 (2007), pp. 80-145.

[7] M. Kanter, Stable densities under change of scale and total variation inequalities, Ann. Probab. 3 (1975), pp. 697-707.

[8] A. Kuznetsov and J.-C. Pardo, Fluctuations of stable processes and exponential functionals of hypergeometric Lévy processes, Acta Appl. Math. 123 (2013), pp. 113-139.

[9] A. E. Kyprianou and J.-C. Pardo, Continuous state branching processes and selfsimilarity, J. Appl. Probab. 45 (4) (2008), pp. 1140-1160.

[10] A. Lachal, Sur le premier instant de passage de l'intégrale du mouvement brownien, Ann. Inst. Henri Poincaré Probab. Stat. 27 (3) (1991), pp. 385-405.

[11] M. Lefebvre, First-passage densities of a two-dimensional process, SIAM J. Appl. Math. 49 (5) (1989), pp. 1514-1523.

[12] J. Letemplier and T. Simon, On the law of homogeneous stable functionals, arXiv: 1510.07441.

[13] J. Pitman and M. Z. Rácz, Beta-gamma tail asymptotics, Electron. Commun. Probab. 20 (2015), paper no. 84 .

[14] C. Profeta and T. Simon, Persistence of integrated stable processes, Probab. Theory Related. Fields 162 (2015), pp. 463-485.

[15] K. Sato, Lévy Processes and Infinitely Divisible Distributions, Cambridge University Press, Cambridge 1999.

[16] T. Simon, Hitting densities for spectrally positive stable processes, Stochastics 83 (2) (2011), pp. 203-214.

[17] T. Simon, Comparing Fréchet and positive stable laws, Electron. J. Probab. 19 (16) (2014), pp. $1-25$.

Julien Letemplier

Laboratoire Paul Painlevé

Université de Lille 1

F-59655 Villeneuve d'Ascq Cedex

E-mail: ju.letemplier@gmail.com
Thomas Simon

Laboratoire Paul Painlevé

Université de Lille 1

F-59655 Villeneuve d'Ascq Cedex

E-mail: simon@math.univ-lille1.fr

Received on 30.12.2015;

revised version on 10.9.2016 